\section{Extra-mammary Paget's disease rising from a non-invasive rectal adenoma}

To the Editor,

Extra-mammary Paget's disease (EMPD) is a chronic erythematous dermatological condition which can be classified as either primary or secondary. Primary EMPD is thought to arise from skin adnexal apocrine glands, whereas, secondary tends to arise from visceral carcinomas, such as a colorectal or gynaecological source. ${ }^{1,2}$ EMPD developing from a non-invasive colorectal adenoma is incredibly rare, with very few documented cases in the current literature. As such, there is very little guidance on treatment and prognosis, although reports suggest these patients may have better outcomes. ${ }^{3}$ Thus, it is critical for a clinical pathological consensus to avoid over treatment. This case report presents an incidental finding of EMPD in a patient with a rectal tubulo-villous adenoma (TVA).

A 70-year-old woman presented for assessment of mild rectal bleeding in the context of known sigmoid diverticular disease. She had no history of malignancy and her only past history was a previous hysterectomy performed decades prior. She had a family history of colorectal cancer (first degree relative). Her laboratory investigations including full blood count and iron studies were within normal limits.

Her index colonoscopy revealed a $6 \mathrm{~mm}$ pedunculated polyp at the dentate line that was resected with cold snare polypectomy. Histology revealed a TVA with low grade dysplasia. A surveillance procedure performed one year later revealed tumour recurrence at the site of previous resection and a new $12 \mathrm{~mm}$ lesion (Fig. 1A). This, and surrounding tissue were removed with combined cold and hot snare polypectomy. Histology revealed the same findings as the original polyp.

A second surveillance procedure six months later revealed ongoing recurrence with an $8 \mathrm{~mm}$ pedunculated lesion at the dentate line. Cold snare polypectomy was again performed. Given the ongoing recurrence, submucosal fibrosis, and the difficult endoscopic location, she was referred for an endoscopic mucosal resection. A $15 \mathrm{~mm}$ polypoid lesion was completely resected using endoscopic submucosal dissection. Histology revealed a TVA with a focal area of high grade dysplasia that was clear of the inked margin (Fig. 2A-C). Adjacent to the adenoma, there was a small fragment of attached squamous mucosa that showed large atypical cells in a pagetoid spread, containing intracytoplasmic mucin. Immunohistochemistry showed that both the adjacent adenoma and these cells were positive for cytokeratin $(\mathrm{CK}) 7$, CK20, CDX-2 and SATB2, indicating an intestinal phenotype (Fig. 2D-F). Gross cystic disease fluid protein (GCDFP)-15, oestrogen receptor, GATA-3 and mammaglobin were negative.

She was referred for gynaecological and colorectal evaluation. Speculum examination of the vaginal vault did not reveal evidence of anterior extension of disease. Serum tumour markers were negative and contrast enhanced computed tomography of the chest, abdomen and pelvis did not reveal a distant primary tumour. There was no evidence of local invasion seen on pelvic magnetic resonance imaging. Examination under anaesthesia (EUA) revealed subtle macroscopic changes including mild induration and oedema from 7 o'clock to 1 o'clock which were consistent with Paget's (Fig. 1B). The biopsies showed extensive disease, confined to the epithelium, with the same intestinal phenotype as the original case. The final pathological diagnosis was EMPD arising from a rectal adenoma. The patient has been reviewed by a multi-disciplinary team and planned for regular surveillance with repeat EUA and biopsy.

Secondary EMPD usually arises when there is intraepithelial invasion (pagetoid spread) of an underlying or contiguous visceral carcinoma. The most common sites are the lower gastro-intestinal or urogenital tract. Differential diagnoses for the microscopic appearance in these areas include melanoma and pagetoid Bowen's disease $;{ }^{2}$ however, these can readily be differentiated with an appropriate immunohistochemical (IHC) panel. Primary perianal Paget's is rare, whilst secondary Paget's disease makes up to $60 \%$ of perianal cases. ${ }^{4}$ Presentations include itch and irritation of the peri-anal area, as well as ulceration and a palpable mass. ${ }^{5}$

Distinguishing primary from secondary EMPD involves both immunohistochemistry and exclusion of an underlying carcinoma. Immunohistochemically, primary tumours arising from adnexal structures tend to have the following IHC markers: $\mathrm{CK} 7+, \mathrm{CK} 20-, \mathrm{GCDFP}+{ }^{6}$ and may also express HER 2 and androgen receptors. In contrast, secondary EMPD of anorectal origin tends to be CK7-, although this is not specific, and CK20+ ${ }^{7,8}$ can also express a range of antigens dependent on the underlying tumour. Of note CDX2 is a specific lower gastrointestinal adenocarcinoma associated EMPD supportive marker. ${ }^{6}$ However, not all secondary EMPDs follow these patterns of expression, such as certain gynaecological and breast carcinomas. Hence, the diagnosis

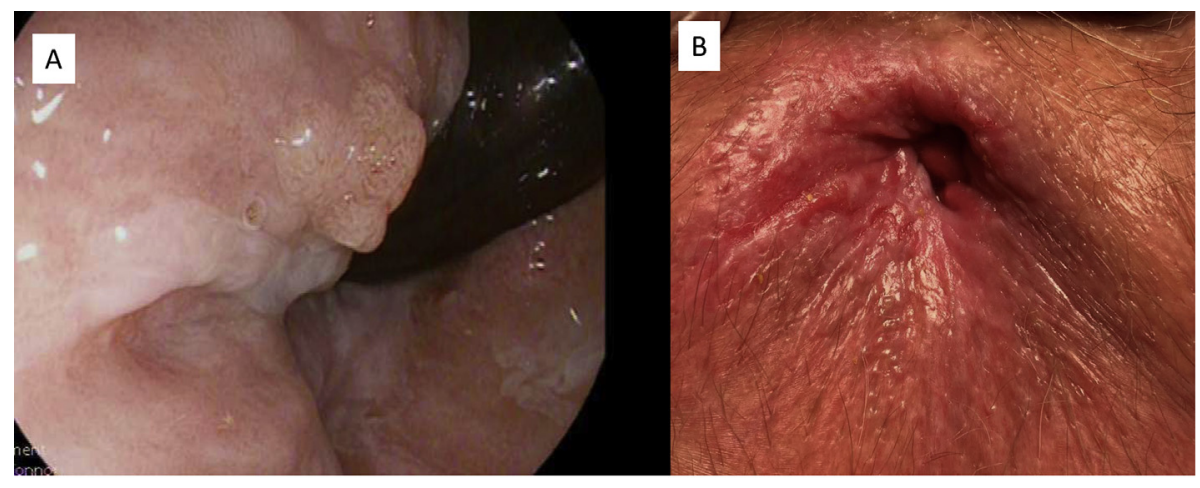

Fig. 1 Macroscopic appearance. (A) Endoscopy, first recurrence. (B) Perianal appearance. 

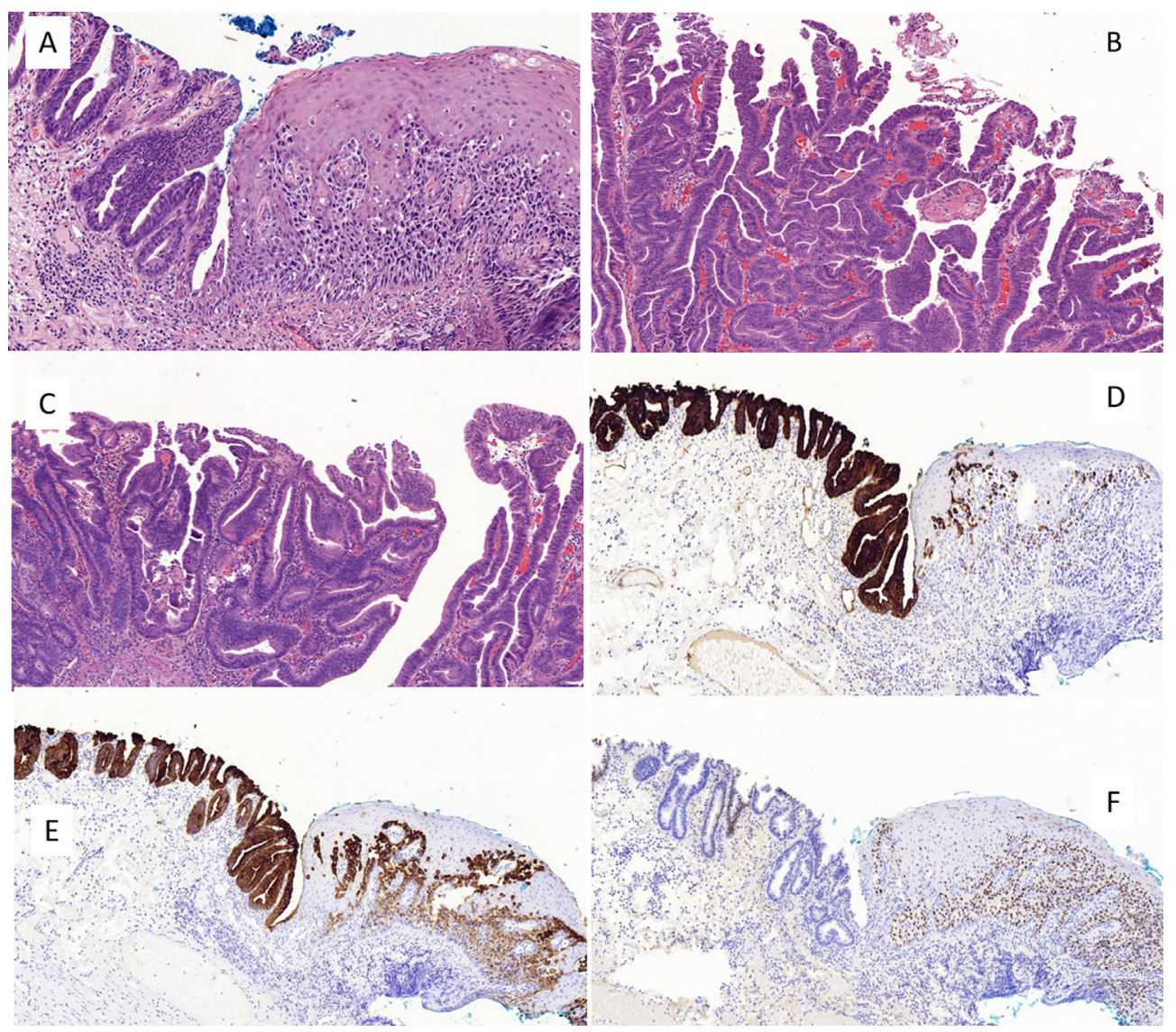

Fig. 2 (A) Non-invasive rectal adenoma with intraepithelial neoplastic cells in the adjacent squamous epithelium (haematoxylin and eosin), with areas of high grade dysplasia (B,C). (D,E,F) Immunohistochemistry shows an intestinal phenotype: (D) CK7, (E) CK20, (F) SATB2 pictured.

needs to be made in conjunction with the clinical findings and exclusion of other causes.

Unlike Paget's disease of the nipple associated with ductal carcinoma in situ, EMPD arising from a non-invasive colorectal adenoma is unusual. Very few cases have been reported in the current literature. In a series of 11 cases of perianal Paget's disease, five of the patients had documented synchronous rectal adenocarcinoma and they were CK7+, CK20+, and GCDFP-. Of the six patients who did not have rectal adenocarcinoma, four were $\mathrm{CK} 7+, \mathrm{CK} 20-$, and GCDFP+. The other two cases were CK7+, CK20+ and GCDFP-, similar to our case. In both cases there was evidence of recurrence after wide local excision, and one was subsequently treated with radiotherapy. ${ }^{9}$ In their case series of 13 patients, Liao and colleagues also found that GCDFP was only positive in patients with primary peri-anal disease, and CDX2 was only positive in secondary disease, whilst CK20 was present in both primary and secondary disease. Eight of the 13 cases also had a synchronous carcinoma, whilst one had an adenoma with high grade dysplasia.

The largest case series to date of EMPD associated with colorectal adenomas published by Hutchings et al. included four cases. Similar to our case, all four had evidence of high grade dysplasia with no evidence of invasion or an invasive adenocarcinoma elsewhere and IHC markers were CK7+, $\mathrm{CK} 20+, \mathrm{GCDFP}-$ and CDX2 +, where available. Only one patient required adjuvant treatment after excision. One had recurrence at 8 months and another was found to have a mucinous adenocarcinoma 36 months after presentation.
In summary, secondary EMPD is a rare condition, especially arising from non-invasive rectal adenomas such as in our case. As such, it is important that pathologists are aware of this condition. Thorough clinical assessment in addition to IHC markers are essential to determine (1) whether these are invasive or non-invasive lesions, and (2) whether these are primary or secondary lesions. Due to the limited number of cases, the optimal treatment strategy following excision of the primary lesion remains uncertain. In any case, after careful assessment and exclusion of a malignant primary, regular surveillance appears to be a reasonable follow-up strategy in order to avoid undue harm from over treatment. However, clinicians need to be aware that recurrence and metachronous malignancy may still occur in these patients.

Conflicts of interest and sources of funding: The authors state that there are no conflicts of interest to disclose.

\section{Harb ${ }^{1,2}$, D. S. Prince ${ }^{1}$, M. Bassan ${ }^{1,2}$, S. Mackenzie Mat, $^{3,4}$} S. J. Connor ${ }^{1,2,5}$, T. Rutland ${ }^{6,7,8}$

${ }^{1}$ Department of Gastroenterology and Hepatology, Liverpool Hospital, Liverpool, NSW, Australia; ${ }^{2}$ South Western Sydney Clinical School, University of NSW, Liverpool, NSW, Australia; ${ }^{3}$ Department of Colorectal Surgery, Liverpool Hospital, Liverpool, NSW, Australia; ${ }^{4}$ Liverpool School of Medicine, Western Sydney University, Liverpool, NSW, Australia; ${ }^{5}$ Ingham Institute for Applied Medical Research, Liverpool, NSW, Australia; ${ }^{6}$ Department of Anatomical 
Pathology, Liverpool Hospital, Liverpool, NSW, Australia; ${ }^{7}$ Discipline of Pathology, School of Medicine, Western Sydney University, Liverpool, NSW, Australia; ${ }^{8}$ Graduate School of Medicine, University of Wollongong, Wollongong, NSW, Australia

Contact Dr Martin Harb.

E-mail: Martin.Harb@health.nsw.gov.au

1. Heymann WR. Extramammary Paget's disease. Clin Dermatol 1993; 11 $83-7$.

2. Shepherd V, Davidson EJ, Davies-Humphreys J. Extramammary Paget's disease. BJOG 2005; 112: 273-9.

3. Hutchings D, Windon A, Assarzadegan N, Salimian KJ, Voltaggio L, Mongtomery EA. Perianal Paget's disease as spread from non-invasive colorectal adenomas. Histopathology 2021; 78: 276-80.

4. Lam C, Funaro D. Extramammary Paget's disease: summary of current knowledge. Dermatol Clin 2010; 28: 807-26.

5. Liao X, Liu X, Fan X, Lai J, Zhang D. Perianal Paget's disease: a clinicopathological and immunohistochemical study of 13 cases. Diagn Pathol 2020; 15: 1-7.

6. De Nisi MC, D'Amuri A, Toscano M, Lalinga AV, Pirtoli L, Miracco C. Usefulness of CDX2 in the diagnosis of extramammary Paget disease associated with malignancies of intestinal type. Br J Dermatol 2005; 153: 677-9.

7. Ohnishi T, Watanabe S. The use of cytokeratins 7 and 20 in the diagnosis of primary and secondary extramammary Paget's disease. Br J Dermatol 2000; 142: 243-7.

8. Battles OE, Page DL, Johnson JE. Cytokeratins, CEA, and mucin histochemistry in the diagnosis and characterization of extramammary Paget's disease. Am J Clin Pathol 1997; 108: 6-12.

9. Goldblum JR, Hart WR. Perianal Paget's disease: a histologic and immunohistochemical study of 11 cases with and without associated rectal adenocarcinoma. Am J Surg Pathol 1998; 22: 170-9.

DOI: https://doi.org/10.1016/j.pathol.2021.11.011

\section{Adenosquamous carcinoma-like mesonephric adenocarcinoma}

\section{To the Editor,}

Mesonephric adenocarcinoma (MNAC) is a rare malignant tumour derived from remnants of the mesonephric duct in the female genital tract. MNAC usually occurs in the cervix, vagina, uterine body and ovary. The histopathological features of MNAC are the combination of multiple growth patterns, showing glandular, tubular and papillary. Due to the rarity of this disease, its aetiology and precursor lesions remain unclear. All MNAC patients reported thus far underwent surgical resection. Adjuvant radiotherapy and chemotherapy are often used in patients with advanced disease, ${ }^{1}$ but the best scheme and effect of treatment are unknown.

Herein, we present a case of a 57-year-old female with vaginal bleeding, less than the menstrual volume, and no abdominal pain. A magnetic resonance imaging (MRI) scan revealed a $3.2 \mathrm{~cm}$ diameter mass in the right anterior wall of the cervix, with high $\mathrm{T} 1$ and $\mathrm{T} 2$ signals; diffusion weighted imaging (DWI) showed high signal intensity, and the arterial phase was significantly intensified after enhancement. U1trasound imaging revealed an irregular hypoechoic area in the cervix, with uneven internal echo and abundant blood flow signals. The first laboratory workup showed an increased CA125 level of $56.29 \mathrm{U} / \mathrm{mL}$. To confirm the diagnosis, biopsy revealed that the tumour was solid and papillary, with a fibrovascular axis, and mimicked a non-keratinising squamous carcinoma. The lesion was misdiagnosed as squamous cell carcinoma (SCC) due to the morphology of the squamous epithelial growth pattern (morphology mimics a high grade squamous intraepithelial lesion) and the lack of immunohistochemistry. As MRI showed that the tumour invaded the parauterine tissue, a course of neoadjuvant therapy (combination of irinotecan and nedaplatin) was carried out prior to surgery of radical hysterectomy and lymphadenectomy. Before the operation, the level of CA125 was reduced to $37.17 \mathrm{U} / \mathrm{mL}$. Grossly, a $2.9 \times 2.9 \times 2.7 \mathrm{~cm}$ ulcerative mass was located in the cervix, invading the upper third of the vagina. The uterine body, ovaries and fallopian tubes were unremarkable. Microscopically, the mass showed a variety of architectural growth patterns, including papillary (Fig. 1A,B), solid and glandular (Fig. 1C,D) patterns. The pattern of papillary growth had fibrovascular axis, and the morphology was similar to that of papillary SCC. Spindlelike epithelial cells could be seen around the glandular structure; the nucleus was round and oval, and the nucleolus was not obvious. Immunohistochemical studies of the tumour cells yielded the following results: negative for CK5/6 (Fig. 2A), oestrogen receptor (ER), progesterone receptor (PR) and p40; diffusely positive for CD10 (Fig. 2B), GATA3 (Fig. 2D), AE1/AE3 and CK7, and focally positive for P16 (Fig. 2C). No metastasis was found in any of the lymph nodes. The patient received postoperative chemotherapy (combination of irinotecan and nedaplatin) and had no evidence of disease 10 months after the operation.

To the best of our knowledge, this is the first report of adenosquamous carcinoma-like MNAC. Mesonephric duct remnants in the cervix are found in approximately $22 \%$ of adult females. ${ }^{2}$ However, tumours from mesonephric remnants rarely occur in the female reproductive system. The diagnosis of MNAC is difficult and challenging, especially for frozen sections and biopsy specimens. Various morphological patterns can be seen in MNAC, such as glandular, papillary and reticular patterns. The histological features of our case were solid and squamous epithelial growth patterns. Initially, the tumour was misdiagnosed as SCC due to the absence of immunohistochemistry analysis. As MRI showed that the tumour invaded the parauterine tissue, we used next generation sequencing (NGS) technology to detect the paraffin tissue of patients for follow-up treatment. NGS results showed TERT, MSH2, TSC2, ARHGAP6, AURKA, CTNNA2, CTNNA3, FAT3, FGF4, HCK, LRP1, MUC16, NSD1, SLIT2 and SLX4 mutations in the tumour tissue. According to the report of targeted genomic analysis using NGS, most MNACs harbour KRAS mutations, ${ }^{3,4}$ suggesting that $K R A S$ mutations are related to the development of MNAC. The histological features of MNAC with KRAS mutations were adenoid, papillary, cribriform and solid. ${ }^{5}$ Although the morphology of this case also shows tubular, solid and papillary structures, the pattern of papillary growth is similar to papillary SCC. TERT mutations have been reported in $70 \%$ of basal cell carcinomas, $50 \%$ of cutaneous SCC, ${ }^{6} 22 \%$ of cervical SCC and $60 \%$ of oral SCC. ${ }^{7}$ In addition, $80 \%$ of SCC of the urinary bladder ${ }^{8}$ also have TERT mutations. In spindle cell carcinoma and spindle cell or spindle cell/squamous cell differentiated breast carcinoma, TERT mutations are as high as $50 \%$. $^{9}$ It could be concluded that the morphology of this case may be related to TERT mutations.

In summary, cervical MNAC is a rare tumour. Cervical lesions are usually detected by biopsy. Because of the 